Article

\title{
Polyhexamethylene Guanidine Phosphate Damages Tight Junctions and the F-Actin Architecture by Activating Calpain-1 via the P2RX7/Ca2+ Signaling Pathway
}

\author{
Sun Woo Jin ${ }^{\dagger}$, Gi Ho Lee ${ }^{\dagger}$, Hoa Thi Pham, Jae Ho Choi and Hye Gwang Jeong *(D) \\ College of Pharmacy, Chungnam National University, Daejeon 34134, Korea; mpassword@cnu.ac.kr (S.W.J.); \\ ghk1900@cnu.ac.kr (G.H.L.); hoapt@cnu.ac.kr (H.T.P.); choijh1@cnu.ac.kr (J.H.C.) \\ * Correspondence: hgjeong@cnu.ac.kr; Tel.: +82-42-821-5936 \\ + These authors contributed equally to this work.
}

Received: 14 November 2019; Accepted: 22 December 2019; Published: 24 December 2019

check for updates

\begin{abstract}
Polyhexamethylene guanidine phosphate (PHMG-p), a member of the polymeric guanidine family, has strong antimicrobial activity and may increase the risk of inflammation-associated pulmonary fibrosis. However, the effect of PHMG-p on the barrier function of the bronchial epithelium is unknown. Epithelial barrier functioning is maintained by tight junctions (TJs); damage to these TJs is the major cause of epithelial barrier breakdown during lung inflammation. The present study showed that, in BEAS-2B human bronchial epithelial cells, exposure to PHMG-p reduced the number of TJs and the E-cadherin level and impaired the integrity of the F-actin architecture. Furthermore, exposure to PHMG-p stimulated the calcium-dependent protease calpain-1, which breaks down TJs. However, treatment with the calpain-1 inhibitor, ALLN, reversed the PHMG-p-mediated impairment of TJs and the F-actin architecture. Furthermore, exposure to PHMG-p increased the intracellular $\mathrm{Ca}^{2+}$ level via P2X purinoreceptor 7 (P2RX7) and inhibition of P2RX7 abolished the PHMG-p-induced calpain-1 activity and protein degradation and increased the intracellular $\mathrm{Ca}^{2+}$ level. Although exposure to PHMG-p increased the extracellular ATP level, hydrolysis of extracellular ATP by apyrase did not influence its detrimental effect on bronchial epithelial cells. These results implicate the impairment of TJs and the F-actin architecture in the pathogenesis of pulmonary diseases.
\end{abstract}

Keywords: PHMG-p; tight junctions; calpain; F-actin; P2RX7

\section{Introduction}

The respiratory epithelial layer protects the surface of the airways and alveoli by trapping and removing infectious particles and airborne pollutants [1]. It is also implicated in a variety of lung diseases, such as asthma and chronic obstructive pulmonary disease, through regulation of the immune response to inflammatory stimuli and promotion of susceptibility to respiratory distress syndrome and lung damage [2,3]. Destruction of the epithelial barrier is a hallmark of respiratory distress syndrome and is confirmed by the presence of high-molecular-weight serum proteins in bronchoalveolar lavage fluid [4].

The epithelial lining of the respiratory tract consists of a monolayer of polarized epithelial cells linked to their neighbors by tight junctions (TJs), adherens junctions (AJs), desmosomes, and gap junctions [5]. TJs and AJs, the most apically located junctions, are collectively referred to as the apical junctional complex, which plays a key role in the formation and maintenance of the epithelial barrier. TJs and AJs consist of transmembrane and peripheral membrane protein complexes [6,7]. Occludin, claudin, and junction adhesion molecules are transmembrane TJ proteins in epithelial cells; these 
proteins seal the paracellular space and regulate paracellular transport between epithelial cells [8]. In addition, adaptor proteins in the cytoplasm, such as zonula occludens (ZO) proteins, support TJ structures to form a scaffold between the transmembrane proteins and the actin cytoskeleton [8]. AJs in epithelial cells are composed of E-cadherin and proteins of the nectin family [5,6]. ZO proteins, the most extensively studied component of $\mathrm{TJ}$ cytoplasmic plaques, mediate interactions between different types of TJs and/or connect them to the actin cytoskeleton [6,7]. The armadillo-family proteins, $\beta$-catenin and p120 catenin, are present in the cytoplasmic plaque of AJs and mediate the interaction of E-cadherin with actin-binding proteins, which link E-cadherin to the actin cytoskeleton [6,7]. Rapid apical junctional complex disassembly is associated with the reorganization of actin filaments by F-actin cytoskeleton-disrupting toxins and inflammatory stimuli $[9,10]$.

Polyhexamethylene guanidine (PHMG) is a guanidine-based polymeric antimicrobial agent [11-13]. The major two derivatives of its salt, PHMG phosphate (PHMG-p) and PHMG hydrochloride, are widely used in detergents, paints, and swimming pools [14-16]. The main antimicrobial mechanism of guanidine derivatives is the destruction of the plasma membrane through the interaction of the cationic nitrogen with negatively charged cell-surface phospholipids, especially phosphatidylglycerol, the main component of the bacterial membrane [17-20]. The cytotoxicity of guanidine derivatives is also mediated by the destruction of the plasma membrane [12,13]. However, the effect of PHMG on TJs is unclear. Therefore, we evaluated the effect of PHMG on TJs and the F-actin architecture, and characterized the underlying mechanism.

\section{Materials and Methods}

\subsection{Chemicals and Reagents}

Dulbecco's modified Eagle's medium/Ham's F-12 Nutrient Mixture (DMEM/F-12), fetal bovine serum, and trypsin-ethylenediaminetetraacetic acid were purchased from Welgene (Gyeongsan, South Korea). 3-(4,5-Dimethylthiazol-2-yl)-2,5-diphenyltetrazolium bromide (MTT) was purchased from USB Corporation (Cleveland, OH, USA). The lactate dehydrogenase (LDH) assay kit was purchased from Roche Applied Science (Indianapolis, IN, USA). Fluo-4 NW and Alexa Fluor 488-conjugated secondary antibodies were purchased from Invitrogen (Carlsbad, CA, USA). Antibodies against ZO-1, E-cadherin, occludin, claudin-1, and calpain-1, as well as horseradish peroxidase-conjugated anti-mouse and anti-rabbit IgG, were purchased from Cell Signaling Technology (Beverly, MA, USA). The anti- $\beta$-actin antibody was purchased from Santa Cruz Biotechnology (Dallas, TX, USA). The enhanced chemiluminescence system was obtained from BioFact (Daejeon, South Korea), and nitrocellulose membranes were purchased from Amersham Pharmacia Biotech (Uppsala, Sweden). Phalloidin, MG 132, ALLN, thapsigargin, and BAPTA-AM were purchased from Sigma-Aldrich (St. Louis, MO, USA). Suramin and A438079 were obtained from Tocris Bioscience (Minneapolis, MN, USA). The Highly Stable ATP Bioluminescence Assay Kit and Calpain Activity Fluorometric Assay Kit were purchased from BioVision (Milpitas, CA, USA). All other chemicals were of the highest grade commercially available.

\subsection{Cell Culture}

BEAS-2B cells were obtained from the American Type Culture Collection (Bethesda, MD, USA). The cells were grown in DMEM/F-12 supplemented with $10 \%$ fetal bovine serum, $100 \mathrm{U} / \mathrm{mL}$ penicillin, and $100 \mu \mathrm{g} / \mathrm{mL}$ streptomycin (HyClone, Logan, UT, USA) at $37^{\circ} \mathrm{C}$ in a humidified incubator with an atmosphere containing $5 \% \mathrm{CO}_{2}$.

\subsection{Cell Viability Assay}

Conventional MTT reduction and LDH assays were used to determine the toxicity of PHMG-p to BEAS-2B cells. Cells were seeded in 48-well plates and allowed to attach overnight. On the following day, the cells were treated with PHMG-p for 4-24 h. MTT solution was added, followed by incubation 
for $30 \mathrm{~min}$, and formazan crystals were solubilized by adding DMSO. The absorbance at $550 \mathrm{~nm}$ was measured using a BioTek Synergy HT microplate reader (BioTek Instruments, Winooski, VT, USA). The medium was collected for LDH assays, and the absorbance was measured at $490 \mathrm{~nm}$ using a BioTek Synergy HT microplate reader. Cell viability (\%) and cytotoxicity (fold-change) were quantified based on the absorbance of treated cells relative to control cells.

\subsection{Immunofluorescence}

Cells were grown on glass coverslips to $60-80 \%$ confluence, fixed in $4 \%$ paraformaldehyde for $20 \mathrm{~min}$ at room temperature, and blocked in 5\% serum for $30 \mathrm{~min}$. After five washes with phosphate-buffered saline containing $0.2 \%$ Tween, the coverslips were immersed in $0.2 \%$ Triton X-100 in phosphate-buffered saline for $10 \mathrm{~min}$. Thereafter, cells were incubated with a primary antibody against ZO-1 (1:100) at $4{ }^{\circ} \mathrm{C}$ overnight or with phalloidin for $40 \mathrm{~min}$ at room temperature to stain F-actin. After they had been washed, the cells that had been incubated with $\mathrm{ZO}-1$ were subsequently incubated with an Alexa Fluor 488-conjugated secondary antibody for $2 \mathrm{~h}$ at room temperature. Nuclei were stained with 4',6-diamidino-2-phenylindole (1:1000 dilution), and the cells were viewed under an EVOS fluorescence microscope (Life Technologies, Carlsbad, CA, USA).

\subsection{Determination of Intracellular $\mathrm{Ca}^{2+}$ Level}

The intracellular $\mathrm{Ca}^{2+}$ level was measured by Fluo-4 NW staining, in accordance with the manufacturer's instructions. Briefly, cells were transferred to black 96-well plates, incubated with Fluo-4 NW for $30 \mathrm{~min}$ at $37^{\circ} \mathrm{C}$ and subsequently in the dark for $30 \mathrm{~min}$ at $25^{\circ} \mathrm{C}$. The cells were then treated with PHMG-p, and the intracellular $\mathrm{Ca}^{2+}$ concentration was determined at excitation/emission wavelengths of 488/512 nm at $20 \mathrm{~s}$ intervals for 5 min using a BioTek Synergy HT microplate reader. Fluorescence images of selected cells were captured using an EVOS fluorescence microscope.

\subsection{Measurement of Calpain Activity}

Calpain activity was evaluated using the Calpain Activity Fluorometric Assay Kit, in accordance with the manufacturer's instructions. Briefly, cells were lysed in extraction buffer for $30 \mathrm{~min}$ on ice and centrifuged at 10,000 rpm for $1 \mathrm{~min}$. Supernatants were collected and protein concentrations were determined using a protein assay kit (Pro-Measure, Intron Biotechnology, Seongnam, South Korea). Supernatants were incubated with substrate (Ac-LLY-AFC) and reaction buffer for $1 \mathrm{~h}$ at $37^{\circ} \mathrm{C}$ in the dark. Upon cleavage of substrate, the fluorogenic portion was measured at excitation/emission wavelengths of 400/505 nm using a BioTek Synergy HT microplate reader. The results are expressed as relative fold changes from the control group. Additional reactions were performed to compare the control group with $1 \mu \mathrm{g}$ Active Calpain I (BioVision) to the PHMG-p treated group with $20 \mu \mathrm{M}$ Z-LLY-FMK (BioVision), a calpain inhibitor. The results are expressed as relative fold changes in fluorescence units.

\subsection{ATP Assay}

The ATP concentration was measured using the Highly Stable ATP Bioluminescence Assay Kit (Biovision). Briefly, cells were treated with PHMG-p; then, $90 \mu \mathrm{L}$ of luciferin-luciferase assay solution was distributed in the wells of a white 96-well plate and medium (10 $\mu \mathrm{L})$ was added to each well. Luminescence was recorded using a Varioskan luminometer (Thermo Electron, Waltham, MA, USA). The ATP concentration $(\mu \mathrm{M})$ was calculated using a standard curve.

\subsection{Western Blotting}

Cell lysates were prepared in lysis buffer $(120 \mathrm{mM} \mathrm{NaCl}, 40 \mathrm{mM}$ Tris [pH 8], and 0.1\% Nonidet P-40) on ice for $30 \mathrm{~min}$ and centrifuged at 10,000 rpm for $15 \mathrm{~min}$. Supernatants were collected and concentrations were determined at $595 \mathrm{~nm}$ using a protein assay kit. Equal amounts of total cellular 
protein were electrophoresed in 8-15\% sodium dodecyl sulfate-polyacrylamide gel electrophoresis gels and transferred to nitrocellulose membranes. After membranes had been blocked in $5 \%$ skim milk for $1 \mathrm{~h}$, they were incubated with primary antibodies overnight, then with the appropriate horseradish peroxidase-conjugated secondary antibodies. Protein bands were detected using an enhanced chemiluminescence Western blotting detection kit (Biofact). The integrated optical density for the protein band was calculated by Image-J software version 1.42q (NIH, Bethesda, MD, USA), and then the values were normalized to $\beta$-actin level.

\subsection{Statistical Analysis}

All experiments were repeated at least three times. Results are reported as means \pm standard deviations. One-way analysis of variance was used to determine significant differences between treatment groups. The Newman-Keuls test was used for comparisons of three or more groups. $p$-values $<0.01$ were considered statistically significant.

\section{Results}

\subsection{Effect of PHMG-p on TJ Proteins and the F-Actin Architecture in BEAS-2B Cells}

Initially, we determined the concentration range of PHMG-p suitable for BEAS-2B cells by using MTT and LDH assays. The cytotoxicity of PHMG-p increased in a time- and concentration-dependent manner (Figure 1A,B). Exposure to PHMG-p at $>4 \mu \mathrm{g} / \mathrm{mL}$ reduced cell viability by $>50 \%$ and increased LDH release threefold. Thus, $4 \mu \mathrm{g} / \mathrm{mL}$ was the maximum PHMG-p concentration used in this study. We evaluated the expression levels of TJ markers (ZO-1, occludin, and claudin-1) and of an AJ marker (E-cadherin), which also contributed to the epithelial barrier function $[5,6]$. Western blotting showed that exposure to PHMG-p reduced the levels of TJ and AJ proteins in a timeand concentration-dependent manner (Figure 1C,D). Furthermore, immunofluorescence and F-actin staining showed that exposure to PHMG-p altered ZO-1 localization near the cell membrane and reduced the integrity of the F-actin architecture (Figure 1E,F).

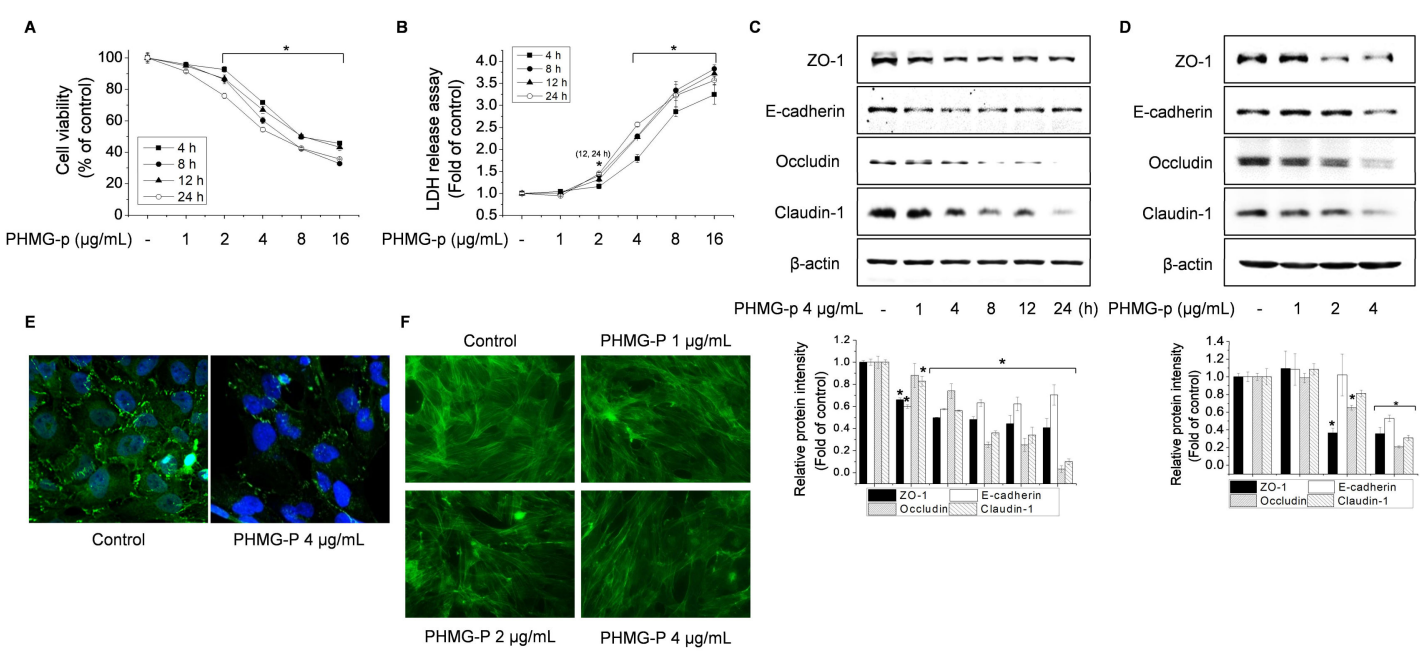

Figure 1. Polyhexamethylene guanidine phosphate (PHMG-p) disrupts the epithelial barrier by degrading tight junction (TJ) proteins and impairing the F-actin architecture in BEAS-2B cells. $(\mathbf{A}, \mathbf{B})$ Cells were treated with 1-16 $\mu \mathrm{g} / \mathrm{mL}$ PHMG-p for 4-24 h. Cytotoxicity was assessed by MTT (A) and LDH (B) assays. Cells were treated with $4 \mu \mathrm{g} / \mathrm{mL}$ PHMG-p for 1-24 h (C) or 1-4 $\mu \mathrm{g} / \mathrm{mL}$ PHMG-p for $4 \mathrm{~h}(\mathbf{D}-\mathbf{F})$, then assessed by western blotting (C,D), immunofluorescence (E), or F-actin staining (F). ${ }^{*} p<0.01$, significantly different from the control. 


\subsection{Role of Calpain in Impairment of TJ Proteins and F-Actin Architecture by PHMG-p}

Intracellular proteases are activated during inflammation and cancer, promoting intracellular cleavage of junction proteins. Sumitomo et al. (2011) demonstrated that streptolysin S from Group A Streptococcus induces calpain activity, leading to the cleavage of occludin and impairment of the barrier function of keratinocytes and intestinal epithelial cells [21]. Wang et al. (2012) showed that activated calpain, following exposure to particulate matter, mediated ZO-1 degradation in human lung microvascular epithelial cells [22]. Accordingly, we investigated the role of calpain in PHMG-p-mediated degradation of TJ proteins. Exposure to PHMG-p increased the level of active calpain-1 and the proteolytic activity of calpain (Figure 2A-C). Furthermore, treatment with the proteasome inhibitor, MG-132, and the calpain-1 inhibitor, ALLN, abolished the PHMG-p-mediated degradation of TJs and the altered F-actin architecture (Figure 2D,E). In addition, Figure S1 showed that ALLN significantly suppressed PHMG-p-reduced cell viability. Taken together, PHMG-p-mediated calpain-1 activation is key event to lead the reduction of cell viability as well as the impairment of tight junctions and the F-actin architecture.
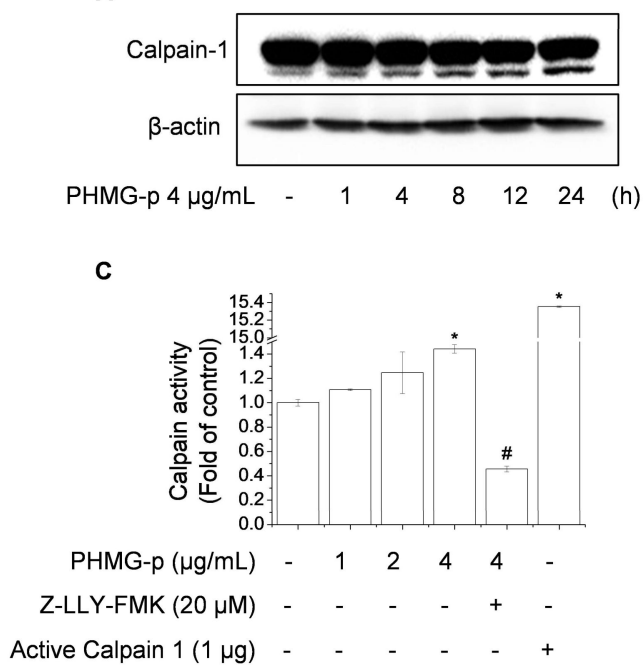

B

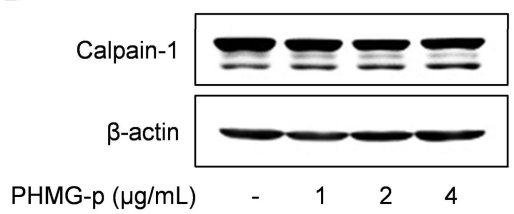

D

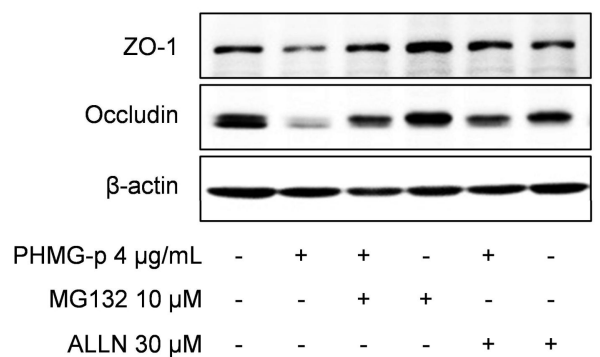

PHMG-P $4 \mu \mathrm{g} / \mathrm{mL}$

E MG132 $10 \mu \mathrm{M} \quad$ ALLN $30 \mu \mathrm{M}$
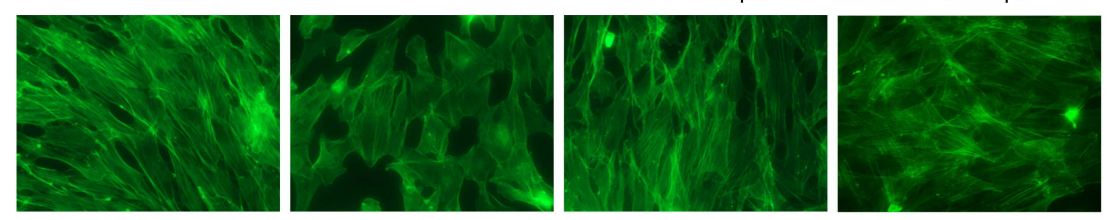

Figure 2. Activated calpain-1 is required for PHMG-p-mediated degradation of tight junctions. Cells were treated with $4 \mu \mathrm{g} / \mathrm{mL}$ PHMG-p for 1-24 h (A) or 1-4 $\mu \mathrm{g} / \mathrm{mL}$ PHMG-p for $4 \mathrm{~h}$ (B), then assessed by western blotting. (C) Cells were treated with $1-4 \mu \mathrm{g} / \mathrm{mL}$ with or without active calpain-1 (positive control) and Z-LLY-FMK (calpain inhibitor), then subjected to calpain activity assay. (D,E) Cells were treated with $10 \mu \mathrm{M}$ MG132 or $30 \mu \mathrm{M}$ ALLN, followed by incubation with $4 \mu \mathrm{g} / \mathrm{mL}$ PHMG-p for $4 \mathrm{~h}$, then assessed by western blotting (D) or F-actin staining (E). ${ }^{*} p<0.01$, significantly different from the control. \#p < 0.01, significantly different from PHMG-p-treated cells.

\subsection{PHMG-p Induces Intracellular $\mathrm{Ca}^{2+}$ Influx via P2RX7}

Calpains are calcium-activated cysteine proteases that exist as various isoforms (e.g., $\mu$ - and m-calpain or calpain-1 and -2). We investigated whether exposure to PHMG-p induces intracellular $\mathrm{Ca}^{2+}$ influx. Indeed, PHMG-p induced intracellular Ca2+ influx, which could be blocked by using ethylenediaminetetraacetic acid (Figure 3A,B). However, thapsigargin, an inhibitor of sarco/endoplasmic 
reticulum $\mathrm{Ca}^{2+}$-ATPase, did not affect the PHMG-p-induced increase in the intracellular $\mathrm{Ca}^{2+}$ level (Figure 3B). These results suggest that the source of $\mathrm{Ca}^{2+}$ is extracellular.

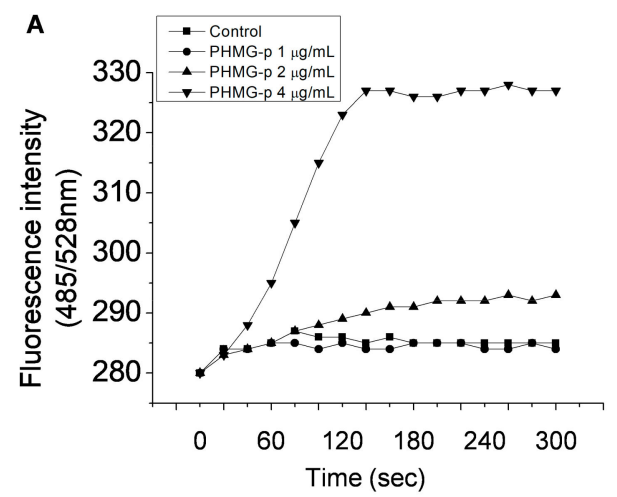

C

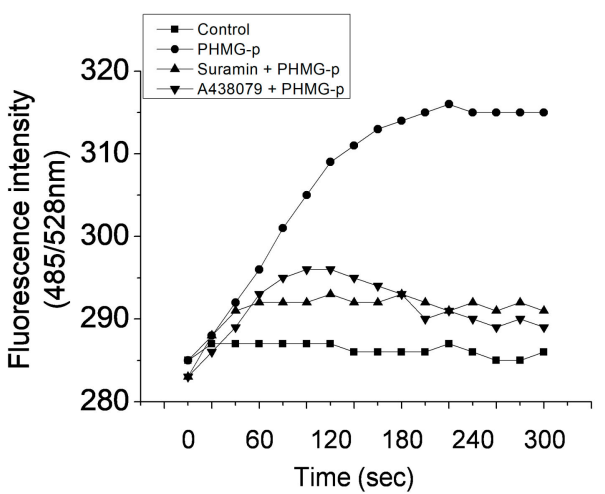

B

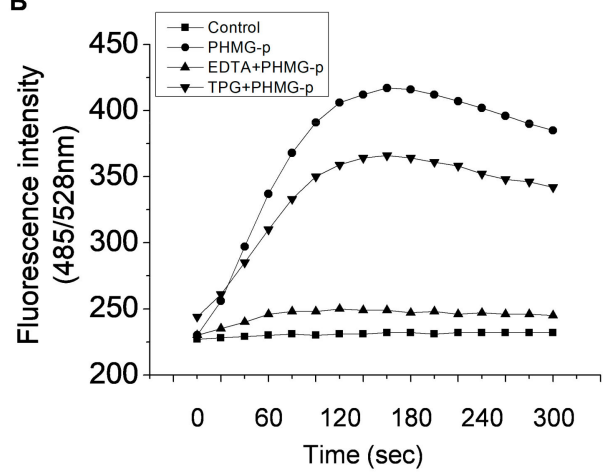

D

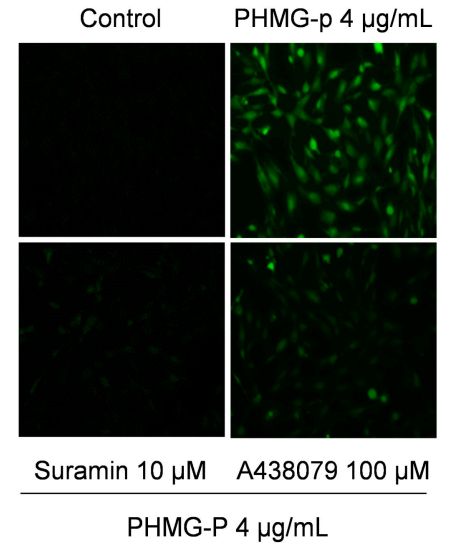

Figure 3. PHMG-p induces intracellular $\mathrm{Ca}^{2+}$ influx via P2RX7. Cells were treated with $1-4 \mu \mathrm{g} / \mathrm{mL}$ PHMG-p (A); $0.5 \mathrm{mM}$ ethylenediaminetetraacetic acid and $1 \mu \mathrm{M}$ thapsigargin, followed by incubation with $4 \mu \mathrm{g} / \mathrm{mL}$ PHMG-p (B); or $10 \mu \mathrm{M}$ suramin and $100 \mu \mathrm{M}$ A438079, followed by $4 \mu \mathrm{g} / \mathrm{mL}$ PHMG-p (C,D). Cells were assessed by Fluo-4 NW staining at 20-s intervals for $5 \mathrm{~min}(\mathbf{A}-\mathbf{C})$ and visualized by fluorescence microscopy (D).

The P2RX7 receptor, a member of the purinergic type-2 receptor family, is a ligand-gated ion channel. P2RX7 signaling plays an important role in $\mathrm{Ca}^{2+}$-related signaling pathways in the epithelium of the airways and alveoli [23]. Song et al. (2016) reported that several purinergic receptors are expressed in BEAS-2B cells [24]. Therefore, we investigated whether P2RX7 is involved in intracellular $\mathrm{Ca}^{2+}$ influx and calpain 1 activation. A general inhibitor of purinergic receptors, suramin, and a P2RX7 inhibitor, A438079, reduced the PHMG-p-induced increase in the intracellular $\mathrm{Ca}^{2+}$ level (Figure 3C and D). These results suggested that P2RX7 is a $\mathrm{Ca}^{2+}$ channel targeted by P2RX7.

\subsection{PHMG-p Induces Calpain-1 Activity and Calpain-1-Dependent Epithelial Barrier Dysfunction via $P 2 R X 7 / \mathrm{Ca}^{2+}$}

To determine whether PHMG-induced intracellular $\mathrm{Ca}^{2+}$ influx affects calpain- 1 activation, we investigated the effect of P2RX7 blockade on calpain-1 activity. Treatment with suramin, A438079, and BAPTA-AM suppressed the PHMG-induced activity of calpain (Figure 4A) and the active form calpain-1 (Figure 4B,C). Furthermore, blockade of $\mathrm{Ca}^{2+}$ channels and depletion of $\mathrm{Ca}^{2+}$ reversed the PHMG-p-mediated degradation of TJs (Figure 4B,C) and altered F-actin architecture (Figure 4D). P2RX7 is an ATP-gated cation channel, and its activation by extracellular ATP (eATP) results in a variety of cellular responses, including $\mathrm{Ca}^{2+}$ influx, membrane pore formation, and cytokine production [25]. Accordingly, we investigated whether PHMG-p induces intracellular $\mathrm{Ca}^{2+}$ influx by P2RX7 through an increase the eATP level. PHMG-p increased the eATP level (Figure S2A); however, the hydrolysis of 
eATP by apyrase did not affect PHMG-p-mediated intracellular $\mathrm{Ca}^{2+}$ influx and degradation of TJs (Figure S2B,C). Therefore, the P2RX7-mediated intracellular $\mathrm{Ca}^{2+}$ is required for calpain- 1 activation by PHMG-p, whereas P2RX7 activation by PHMG-p is not involved in the increased eATP level.

A

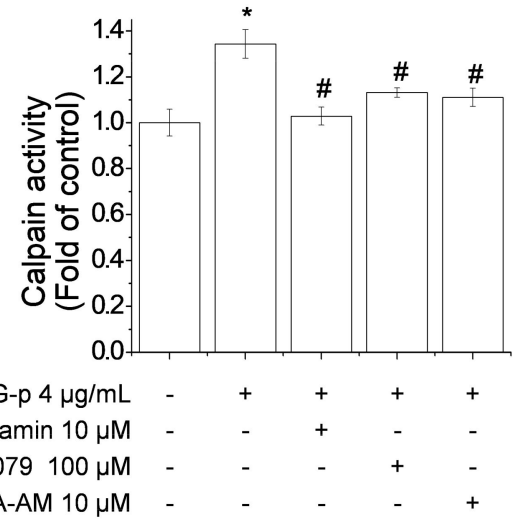

B

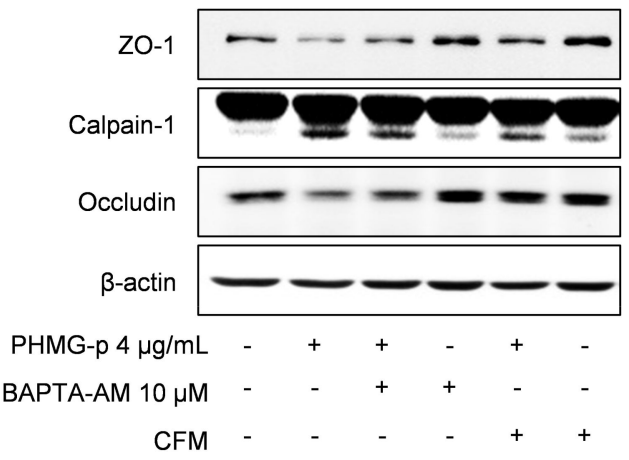

D

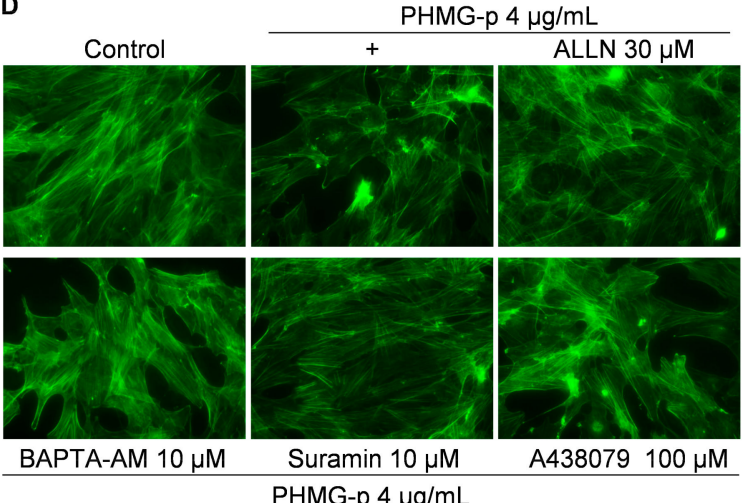

Figure 4. Blockade of PHMG-p-mediated intracellular $\mathrm{Ca}^{2+}$ influx reduces calpain-1 activity and calpain-1-dependent disruption of the epithelial barrier. (A) Cells were treated with or without $10 \mu \mathrm{M}$ suramin, $100 \mu \mathrm{M}$ A438079, and $10 \mu \mathrm{M}$ BAPTA-AM, followed by incubation with $4 \mu \mathrm{g} / \mathrm{mL}$ PHMG-p, then subjected to calpain activity assay. (B,C) Cells were treated with $10 \mu \mathrm{M}$ BAPTA-AM, $10 \mu \mathrm{M}$ suramin, and $100 \mu \mathrm{M}$ A438079, or medium was replaced with $\mathrm{Ca}^{2+}$-free DMEM/F-12 (CFM), followed by incubation with $4 \mu \mathrm{g} / \mathrm{mL}$ PHMG-p and western blotting. (D) Cells were treated with or without $10 \mu \mathrm{M}$ suramin, $100 \mu \mathrm{M}$ A438079, $10 \mu \mathrm{M}$ BAPTA-AM, and $30 \mu \mathrm{M}$ ALLN, followed by incubation with $4 \mu \mathrm{g} / \mathrm{mL}$ PHMG-p and F-actin staining. ${ }^{*} p<0.01$, significantly different from the control. \#p<0.01, significantly different from PHMG-p-treated cells.

\section{Discussion}

We investigated the molecular mechanism by which PHMG-p promotes disruption of the epithelial barrier in BEAS-2B cells. One of our principal findings was that PHMG-p induced the degradation of TJs and impairment of F-actin integrity by activation of calpain-1. Furthermore, PHMG-p increased calpain-1 activity via the promotion of intracellular Ca2 influx through P2RX7, which was essential for its effect on TJ proteins and the F-actin architecture.

TJs in the lung epithelium play a key role in barrier function and form the sealing interface between adjacent epithelial cells [26]. During lung inflammation, the destruction of TJ proteins disrupts the epithelial barrier. In this study, we found that PHMG-p reduced the levels of TJ markers (ZO-1, occludin, and claudin-1) and of an AJ marker (E-cadherin) in BEAS-2B cells. Similarly, PHMG-p has been reported to reduce transepithelial electrical resistance in a bronchial ALI co-culture model; notably, transepithelial electrical resistance is involved in the disruption of the airway epithelial barrier [27]. Song et al. (2019) reported that the cationic nature of PHMG-p could disrupt membrane 
pore formation or integrity in lung epithelial cells, fibroblasts, and monocytes [13]. Furthermore, $\mathrm{F}$-actin cytoskeleton-disrupting toxins have been shown to induce rapid $\mathrm{AJ} / \mathrm{TJ}$ disassembly, indicating that the reorganization of actin filaments is responsible for the loss of AJs during inflammation [9]. Inflammatory stimuli reportedly induce F-actin alterations and AJ/TJ disassembly [10]. In this study, exposure to PHMG-p altered F-actin integrity in a concentration-dependent manner, consistent with degradation of apical junctional complex proteins. Therefore, we concluded that PHMG-p may disrupt the epithelial barrier by impairing TJs and the F-actin architecture in pulmonary epithelial cells.

TJ proteins are associated with the underlying actin cytoskeleton through a scaffold protein that constrains their intracellular domains, which can also be targeted by proteases located near the cytoplasmic surface of the plasma membrane [28]. Intracellular and membrane-related proteases, such as calpain, mediate the intracellular cleavage of junction proteins, thereby promoting cytoskeletal alteration [29]. Wang et al. (2012) showed that particulate-matter air pollution could induce calpain-mediated degradation of TJ proteins and disrupt the endothelial barrier [22]. Heijink et-al. (2012) reported that cigarette smoke extract reduces epithelial integrity by epidermal growth factor receptorand calpain-dependent disruption of intercellular contacts, which may increase susceptibility to environmental insults such as inhaled pathogens [30]. In this study, exposure to PHMG-p was observed to activate calpain- 1 and increase its activity. In addition, the calpain- 1 inhibitor ALLN suppressed PHMG-p-mediated degradation of TJs and alteration of F-actin structure. These results suggest that PHMG-p impairs TJ proteins and F-actin in a calpain-1-dependent manner. Interestingly, inhibition of proteasome activity by MG-132 showed the similar effect of ALLN on PHMG-p-mediated disruption of the epithelial barrier, but further studies are needed to determine how PHMG-p affect degradation of TJs and alteration of F-actin structure via proteasome activity.

P2RX7 receptor hyperactivation is implicated in the pathogenesis of a variety of respiratory diseases, including pulmonary fibrosis, acute lung injury, asthma, pulmonary hypertension, and chronic obstructive pulmonary disease [31,32]. Treatment with A438079, a highly selective antagonist of P2RX7, attenuated hyperpermeability in a model of the human blood-brain barrier and suppressed the ZO-1 and occludin levels [33]. In addition, Wesslau et al. (2019) reported a robust increase in epithelial junction adhesion molecule-A expression in P2RX7-knockout mice, compared to wild-type mice; moreover, they reported that inhibition of P2RX7 by oxATP increased the junction adhesion molecule-A protein level [34]. In the present study, we found that exposure to PHMG-p induced intracellular $\mathrm{Ca}^{2+}$ influx via P2RX7; furthermore, the inhibition of P2RX7 by A438079 suppressed the PHMG-p-mediated degradation of TJs and altered F-actin structure by reducing $\mathrm{Ca}^{2+}$-dependent calpain-1 activity. The P2RX7 signaling pathway is involved in NLRP3 inflammasome activation, and our data are consistent with the findings of a previous report, in which PHMG-p induced lung fibrosis and pulmonary inflammation through activation of the NLRP3 inflammasome in mouse lung tissue [35]. Although ATP is a selective endogenous ligand of P2RX7, and it has low affinity for ATP; thus, high levels of eATP (in the millimolar range) are required for P2RX7-dependent cellular responses in vitro $[25,36]$. In the present study, exposure to PHMG-p in the nanomolar range increased the eATP level, while eATP hydrolysis by apyrase did not affect PHMG-p-mediated intracellular $\mathrm{Ca}^{2+}$ influx, calpain-1 activation, or TJ degradation. P2RX7 can be activated by structurally unrelated agents, such as the antibiotic, polymyxin B [37]; the bactericidal peptide, LL-37 [38]; an amyloidogenic $\beta$ peptide [39]; and serum amyloid A [40]. Other agents, such as Alu-RNA, have been suggested to activate P2RX7 by acting on the intracellular N- or C-terminal domains [41]. Therefore, the PHMG-p-induced increase in eATP might not be involved in the activation of P2RX7.

\section{Conclusions}

In conclusion, exposure to PHMG-p induced the degradation of TJ proteins and impairment of F-actin structural integrity by stimulating the proteolytic activity of calpain-1. PHMG-p-mediated calpain-1 activity promoted intracellular $\mathrm{Ca}^{2+}$ influx via ATP-independent activation of P2RX7. These 
results suggest that PHMG-p-mediated activation of calpain-1 via the P2RX7/Ca ${ }^{2+}$ signaling pathway could induce pulmonary disease by impairing the barrier function of the bronchial epithelium (Figure 5).

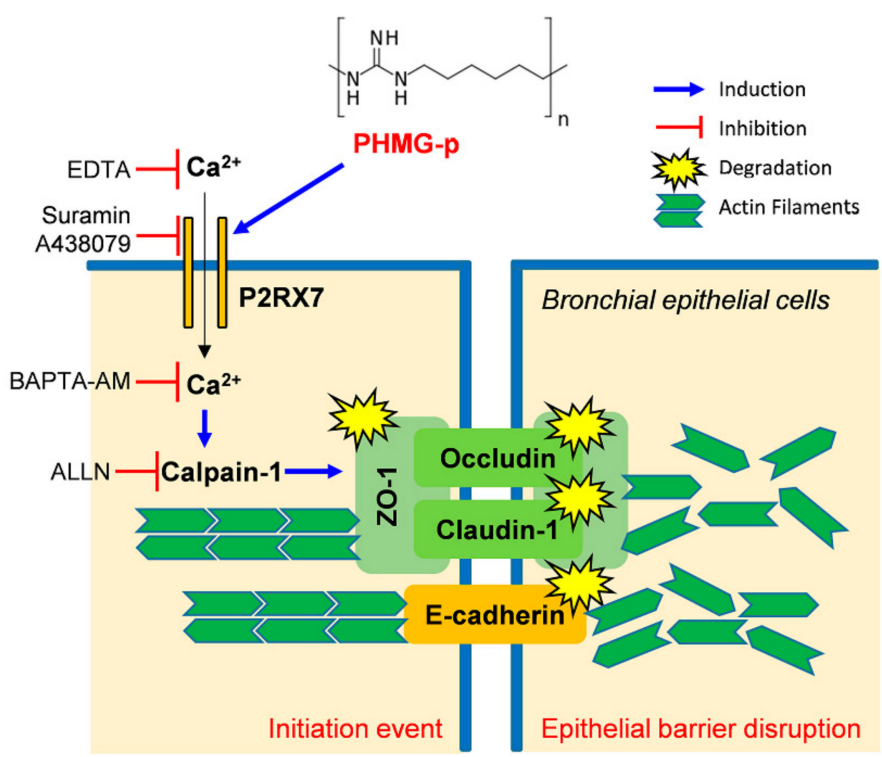

Figure 5. Schematic diagram illustrating the mechanism by which PHMG-p induced the degradation of TJ proteins and impairment of F-actin structural integrity by stimulating the proteolytic activity of calpain-1 via the P2RX7/Ca2+ signaling pathway.

Supplementary Materials: The following are available online at http://www.mdpi.com/2073-4409/9/1/59/s1, Figure S1: PHMG-p decreases cell viability by calapin-1 activation; Figure S2: PHMG-p induces ATP-independent activation of P2RX7.

Author Contributions: Conceptualization, supervision, project managing, funding acquisition: H.G.J. Methodology: S.W.J., and G.H.L. Data analysis: S.W.J., G.H.L., and H.T.P. Experiments: S.W.J., G.H.L., H.T.P., and J.H.C. Draft writing: H.G.J., and S.W.J. Article correction: H.G.J., S.W.J., and J.H.C. All authors have read and agreed to the published version of the manuscript.

Funding: This study was funded by the Korea Ministry of Environment (MOE) as “the Environmental Health Action Program" (2017001360001).

Conflicts of Interest: The authors declare no conflict of interest.

\section{Abbreviations}

AJs adherens junctions

DMEM/F-12 Dulbecco's modified Eagle's medium/Ham's F-12 Nutrient Mixture

eATP extracellular ATP

$\mathrm{LDH} \quad$ lactate dehydrogenase

MTT 3-(4,5-Dimethylthiazol-2-yl)-2,5-diphenyltetrazolium bromide

PHMG-p polyhexamethylene guanidine phosphate

P2RX7 P2X purinoreceptor 7

TJs tight junctions

ZO zonula occludens

\section{References}

1. Wittekindt, O.H. Tight junctions in pulmonary epithelia during lung inflammation. Pflug. Arch. 2017, 469, 135-147. [CrossRef] [PubMed] 
2. Cromwell, O.; Hamid, Q.; Corrigan, C.J.; Barkans, J.; Meng, Q.; Collins, P.D.; Kay, A.B. Expression and generation of interleukin-8, IL-6 and granulocyte-macrophage colony-stimulating factor by bronchial epithelial cells and enhancement by IL-1 beta and tumour necrosis factor-alpha. Immunology 1992, 77, 330-337. [PubMed]

3. Ware, L.B.; Matthay, M.A. The acute respiratory distress syndrome. N. Engl. J. Med. 2000, 342, $1334-1349$. [CrossRef] [PubMed]

4. Holter, J.F.; Weiland, J.E.; Pacht, E.R.; Gadek, J.E.; Davis, W.B. Protein permeability in the adult respiratory distress syndrome loss of size selectivity of the alveolar epithelium. J. Clin. Investig. 1986, 78, 1513-1522. [CrossRef] [PubMed]

5. Tsukita, S.; Furuse, M.; Itoh, M. Multifunctional strands in tight junctions. Nat. Rev. Mol. Cell Biol. 2001, 2, 285-293. [CrossRef] [PubMed]

6. Hartsock, A.; Nelson, W.J. Adherens and tight junctions: Structure, function and connections to the actin cytoskeleton. Biochim. Biophys. Acta Biomembr. 2008, 1778, 660-669. [CrossRef] [PubMed]

7. Pokutta, S.; Weis, W.I. Structure and mechanism of cadherins and catenins in cell-cell contacts. Annu. Rev. Cell Dev. Biol. 2007, 23, 237-261. [CrossRef]

8. Chiba, H.; Osanai, M.; Murata, M.; Kojima, T.; Sawada, N. Transmembrane proteins of tight junctions. Biochim. Biophys. Acta. 2008, 1778, 588-600. [CrossRef]

9. Madara, J.L.; Moore, R.; Carlson, S. Alteration of intestinal tight junction structure and permeability by cytoskeletal contraction. Am. J. Physiol. 1987, 253, 854-861. [CrossRef] [PubMed]

10. Ivanov, A.I. Actin motors that drive formation and disassembly of epithelial apical junctions. Front. Biosci. 2008, 13, 6662-6681. [CrossRef] [PubMed]

11. Kim, H.R.; Hwang, G.W.; Naganuma, A.; Chung, K.H. Adverse health effects of humidifier disinfectants in Korea: Lung toxicity of polyhexamethylene guanidine phosphate. J. Toxicol. Sci. 2016, 41, 711-717. [CrossRef] [PubMed]

12. Park, E.J.; Park, S.J.; Kim, S.; Lee, K.; Chang, J. Lung fibroblasts may play an important role in clearing apoptotic bodies of bronchial epithelial cells generated by exposure to PHMG-P-containing solution. Toxicol. Lett. 2018, 286, 108-119. [CrossRef] [PubMed]

13. Song, J.; Jung, K.J.; Yoon, S.J.; Lee, K.; Kim, B. Polyhexamethyleneguanidine phosphate induces cytotoxicity through disruption of membrane integrity. Toxicology 2019, 414, 35-44. [CrossRef] [PubMed]

14. Ohta, S.; Misawa, Y.; Miyamoto, H.; Makino, M.; Nagai, K.; Shiraishi, T.; Nakagawa, Y.; Yamato, S.; Tachikawa, E.; Zenda, H. A comparative study of characteristics of current-type and conventional-type cationic bactericides. Biol. Pharm. Bull. 2001, 24, 1093-1096. [CrossRef] [PubMed]

15. Oule, M.K.; Azinwi, R.; Bernier, A.M.; Kablan, T.; Maupertuis, A.M.; Mauler, S.; Nevry, R.K.; Dembele, K.; Forbes, L.; Diop, L. Polyhexamethylene guanidine hydrochloride-based disinfectant: A novel tool to fight meticillin-resistant Staphylococcus aureus and nosocomial infections. J. Med. Microbiol. 2008, 57, 1523-1528. [CrossRef] [PubMed]

16. Oule, M.K.; Quinn, K.; Dickman, M.; Bernier, A.M.; Rondeau, S.; DeMoissac, D.B.; Boisvert, A.; Diop, L. Akwaton, polyhexamethylene-guanidine hydrochloride-based sporicidal disinfectant: A novel tool to fight bacterial spores and nosocomial infections. J. Med. Microbiol. 2012, 61, 1421-1427. [CrossRef] [PubMed]

17. Broxton, P.; Woodcock, P.M.; Heatley, F.; Gilbert, P. Interaction of some polyhexamethylene biguanides and membrane phospholipids in Escherichia coli. J. Appl. Bacteriol. 1984, 57, 115-124. [CrossRef]

18. Ikeda, T.; Ledwith, A.; Bamford, C.H.; Hann, R.A. Interaction of a polymeric biguanide biocide with phospholipid membranes. Biochim. Biophys. Acta 1984, 769, 57-66. [CrossRef]

19. Ikeda, T.; Tazuke, S.; Watanabe, M. Interaction of biologically active molecules with phospholipid membranes. I. Fluorescence depolarization studies on the effect of polymeric biocide bearing biguanide groups in the main chain. Biochim. Biophys. Acta 1983, 735, 380-386. [CrossRef]

20. Carmona-Ibeiro, A.M.; de Melo Carrasco, L.D. Cationic antimicrobial polymers and their assemblies. Int. J. Mol. Sci. 2013, 14, 9906-9946. [CrossRef]

21. Sumitomo, T.; Nakata, M.; Higashino, M.; Jin, Y.; Terao, Y.; Fujinaga, Y.; Kawabata, S. Streptolysin S contributes to group A streptococcal translocation across an epithelial barrier. J. Biol. Chem. 2011, 286, 2750-2761. [CrossRef] [PubMed] 
22. Wang, T.; Wang, L.; Moreno-Vinasco, L.; Lang, G.D.; Siegler, J.H.; Mathew, B.; Usatyuk, P.V.; Samet, J.M.; Geyh, A.S.; Breysse, P.N.; et al. Particulate matter air pollution disrupts endothelial cell barrier via calpain-mediated tight junction protein degradation. Part Fibre Toxicol. 2012, 9, 35. [CrossRef] [PubMed]

23. Mishra, A. New insights of P2X7 receptor signaling pathway in alveolar functions. J. Biomed. Sci. 2013, 20, 26. [CrossRef] [PubMed]

24. Song, S.; Jacobson, K.N.; McDermott, K.M.; Reddy, S.P.; Cress, A.E.; Tang, H.; Dudek, S.M.; Black, S.M.; Garcia, J.G.; Makino, A.; et al. Capsaicin-induced Ca2+ signaling is enhanced via upregulated TRPV1 channels in pulmonary artery smooth muscle cells from patients with idiopathic PAH. Am. J. Physiol. Cell Physiol. 2016, 312, 309-325. [CrossRef]

25. Takenouchi, T.; Suzuki, S.; Shinkai, H.; Tsukimoto, M.; Sato, M.; Uenishi, H.; Kitani, H. Extracellular ATP does not induce P2X7 receptor-dependent responses in cultured renal- and liver-derived swine macrophages. Results Immunol. 2014, 4, 62-67. [CrossRef]

26. Schlingmann, B.; Molina, S.A.; Koval, M. Claudins: Gatekeepers of lung epithelial function. Semin. Cell Dev. Biol. 2015, 42, 47-57. [CrossRef]

27. Kim, H.R.; Lee, K.; Park, C.W.; Song, J.A.; Shin, D.Y.; Park, Y.J.; Chung, K.H. Polyhexamethylene guanidine phosphate aerosol particles induce pulmonary inflammatory and fibrotic responses. Arch. Toxicol. 2016, 90, 617-632. [CrossRef]

28. Nava, P.; Kamekura, R.; Nusrat, A. Cleavage of transmembrane junction proteins and their role in regulating epithelial homeostasis. Tissue Barriers 2013, 1, e24783. [CrossRef]

29. Marambaud, P.; Shioi, J.; Serban, G.; Georgakopoulos, A.; Sarner, S.; Nagy, V.; Baki, L.; Wen, P.; Efthimiopoulos, S.; Shao, Z.; et al. A presenilin- $1 / \gamma$-secretase cleavage releases the E-cadherin intracellular domain and regulates disassembly of adherens junctions. EMBO J. 2002, 21, 1948-1956. [CrossRef]

30. Heijink, I.H.; Brandenburg, S.M.; Postma, D.S.; van Oosterhout, A.J. Cigarette smoke impairs airway epithelial barrier function and cell-cell contact recovery. Eur. Respir. J. 2012, 39, 419-428. [CrossRef]

31. Burnstock, G.; Novak, I. Purinergic signalling in the pancreas in health and disease. J. Endocrinol. 2012, 213, 123-141. [CrossRef] [PubMed]

32. Gentile, D.; Natale, M.; Lazzerini, P.E.; Capecchi, P.L.; Laghi-Pasini, F. The role of P2X7 receptors in tissue fibrosis: A brief review. Purinergic Signal. 2015, 11, 435-440. [CrossRef] [PubMed]

33. Yang, F.; Zhao, K.; Zhang, X.; Zhang, J.; Xu, B. ATP Induces Disruption of Tight Junction Proteins via IL-1 Beta-Dependent MMP-9 Activation of Human Blood-Brain Barrier In Vitro. Neural Plast. 2016, 2016, 8928530. [CrossRef] [PubMed]

34. Wesslau, K.P.; Stein, A.; Kasper, M.; Barth, K. P2X7 Receptor Indirectly Regulates the JAM-A Protein Content via Modulation of GSK-3ß. Int. J. Mol. Sci. 2019, 20, 2298. [CrossRef]

35. Kim, H.Y.; Kim, M.S.; Kim, S.H.; Joen, D.; Lee, K. Protective Effects of Nintedanib against Polyhexamethylene Guanidine Phosphate-Induced Lung Fibrosis in Mice. Molecules 2018, 23, 1974. [CrossRef]

36. Surprenant, A.; Rassendren, F.; Kawashima, E.; North, R.A.; Buell, G. The cytolytic P2Z receptor for extracellular ATP identified as a P2X receptor (P2X7). Science 1996, 272, 735-738. [CrossRef]

37. Ferrari, D.; Pizzirani, C.; Gulinelli, S.; Callegari, G.; Chiozzi, P.; Idzko, M.; Panther, E.; Di Virgilio, F. Modulation of $\mathrm{P} 2 \mathrm{X} 7$ receptor functions by polymyxin B: Crucial role of the hydrophobic tail of the antibiotic molecule. Br. J. Pharmacol. 2007, 150, 445-454. [CrossRef]

38. Elssner, A.; Duncan, M.; Gavrilin, M.; Wewers, M.D. A novel P2X7 receptor activator, the human cathelicidin-derived peptide LL37, induces IL-1 beta processing and release. J. Immunol. 2004, 172, 4987-4994. [CrossRef]

39. Sanz, J.M.; Chiozzi, P.; Ferrari, D.; Colaianna, M.; Idzko, M.; Falzoni, S.; Fellin, R.; Trabace, L.; Di Virgilio, F. Activation of microglia by amyloid \{beta\} requires P2X7 receptor expression. J. Immunol. 2009, 182, 4378-4385. [CrossRef] 
40. Niemi, K.; Teirilä, L.; Lappalainen, J.; Rajamäki, K.; Baumann, M.H.; Öörni, K.; Wolff, H.; Kovanen, P.T.; Matikainen, S.; Eklund, K.K. Serum amyloid A activates the NLRP3 inflammasome via P2X7 receptor and a cathepsin B-sensitive pathway. J. Immunol. 2011, 186, 6119-6128. [CrossRef]

41. Fowler, B.J.; Gelfand, B.D.; Kim, Y.; Kerur, N.; Tarallo, V.; Hirano, Y.; Amarnath, S.; Fowler, D.H.; Radwan, M.; Young, M.T.; et al. Nucleoside reverse transcriptase inhibitors possess intrinsic anti-inflammatory activity. Science 2014, 346, 1000-1003. [CrossRef] [PubMed] 